\title{
Sklyanin Determinant for Reflection Algebra
}

Natasha ROZHKOVSKAYA

Department of Mathematics, Kansas State University, USA

E-mail: rozhkovs@math.ksu.edu

Received September 21, 2010, in final form December 23, 2010; Published online December 29, 2010 doi:10.3842/SIGMA.2010.100

\begin{abstract}
Reflection algebras is a class of algebras associated with integrable models with boundaries. The coefficients of Sklyanin determinant generate the center of the reflection algebra. We give a combinatorial description of Sklyanin determinant suitable for explicit computations.
\end{abstract}

Key words: reflection equation; Sklyanin determinant

2010 Mathematics Subject Classification: 05E10; 17B37

In [7] E.K. Sklyanin introduced a class of algebras associated with integrable models with boundaries. Following [5], we call them reflection algebras. The family of reflection algebras $\mathcal{B}(n, l)$ is defined as associative algebras whose generators satisfy two types of relations: the reflection equation and the unitary condition. In [5] A.I. Molev and E. Ragoucy show that the center of $\mathcal{B}(n, l)$ is generated by the coefficients of an analogue of quantum determinant, called the Sklyanin determinant. In the same paper the authors develop an analogue of Drinfeld's highest weight theory for reflection algebras and give a complete description of their finitedimensional irreducible representations.

The reflection algebras $\mathcal{B}(n, l)$ have many common features with the twisted Yangians, introduced by G. Olshanski [6]. For example, the center of twisted Yangian is also generated by coefficients of Sklyanin determinant, which is defined similarly to the Sklyanin determinant of reflection algebras. The detailed exposition of the representation theory of twisted Yangians can be found in $[1,2]$.

In [3] A.I. Molev gives a combinatorial formula for the Sklyanin determinant of twisted Yangians in terms of matrix elements of the matrix of generators of the twisted Yangians (see also [1]). The goal of this paper is to give analogues combinatorial description of the Sklaynin determinant of the reflection algebra.

In Section 1 we review the main definitions. In Sections 2, 3 we rewrite the definition of the Sklyanin determinant of $\mathcal{B}(n, l)$ in an alternative form. We describe combinatorics of the corresponding product of generating matrices 'twisted' by Jucys-Murphy elements. In Section 4, Theorem 1, we prove combinatorial formula for Sklyanin determinant in terms of matrix elements of the generating matrix of $\mathcal{B}(n, l)$. In Section 5 we give examples of computations by this formula.

\section{Definitions}

The following notations will be used through the paper. For a matrix $X$ with entries $\left(x_{i j}\right)_{i, j=1, \ldots, n}$ in an associative algebra $A$ write

$$
X_{s}=\sum_{i, j} 1 \otimes \cdots \otimes E_{i j} \otimes \cdots \otimes 1 \otimes x_{i j} \in \operatorname{End}\left(\mathbb{C}^{n}\right)^{\otimes k} \otimes A .
$$

\footnotetext{
* This paper is a contribution to the Proceedings of the International Workshop "Recent Advances in Quantum Integrable Systems". The full collection is available at http://www.emis.de/journals/SIGMA/RAQIS2010.html
} 
Consider the Yang matrix

$$
R(u)=1-\frac{P}{u} \in \operatorname{End}\left(\mathbb{C}^{n}\right)^{\otimes 2}\left[\left[u^{-1}\right]\right],
$$

where $P: v \otimes w \rightarrow w \otimes v$ is a permutation operator in the space $\mathbb{C}^{n} \otimes \mathbb{C}^{n}$.

For $i, j$ such that $1 \leq i<j \leq k$ the notations $P_{i j}$ and $R_{i j}(u)$ are used for the action $P$ and $R(u)$ on the $i$-th and $j$-th copies of the vector space $\mathbb{C}^{n}$ in $\left(\mathbb{C}^{n}\right)^{\otimes k}$.

Let $m, l$ be such nonnegative integers that $n=m+l$. Let $\left(\varepsilon_{1}, \ldots, \varepsilon_{n}\right)$ be an $n$-tuple with entries \pm 1 , such that

$$
\varepsilon_{i}=\left\{\begin{array}{rll}
1 & \text { if } & 1 \leq i \leq m \\
-1 & \text { if } & n \geq i>m .
\end{array}\right.
$$

The reflection algebra $\mathcal{B}(n, l)$ is a unital associative algebra with the generators $b_{i j}^{(r)}, i, j=$ $1, \ldots, n, r=0,1,2, \ldots$, that satisfy the relations of the reflection equation (1) and the unitary condition (2) described below.

Combine the generators $b_{i j}^{(r)}$ into formal series

$$
b_{i}^{j}(u)=\sum_{r=0}^{\infty} b_{i j}^{(r)} u^{-r} \quad \text { with } \quad b_{i j}^{(0)}=\delta_{i j} \varepsilon_{i},
$$

and collect them into generating matrix

$$
B(u)=\sum_{i, j=1}^{n} E_{i j} \otimes b_{i}^{j}(u) \in \operatorname{End} \mathbb{C}^{n} \otimes \mathcal{B}(n, l)\left[\left[u^{-1}\right]\right] .
$$

Then the reflection equation relation is given by

$$
R(u-v) B_{1}(u) R(u+v) B_{2}(v)=B_{2}(v) R(u+v) B_{1}(u) R(u-v),
$$

and the unitary condition is

$$
B(u) B(-u)=1 .
$$

Remark 1. The unitary condition allows to realize the algebra $\mathcal{B}(n, l)$ as a subalgebra of Yangian $Y\left(\mathfrak{g l}_{n}(\mathbb{C})\right)$. The mentioned below Proposition 1 is proved in [5] through this inclusion of algebras. However, we do not need the unitary conditions anywhere for the proofs of the statements below. Hence all the results of the paper automatically become true for the extended version of the reflection algebra, which is defined the same way as the algebra $\mathcal{B}(n, l)$, but with the unitary condition (2) omitted.

We abbreviate $R_{i j}:=R_{i j}(2 u-i-j+2)$. Put

$$
\left\langle B_{1}, \ldots, B_{k}\right\rangle=B_{1}(u)\left(R_{12} \cdots R_{1 k}\right) B_{2}(u-1)\left(R_{23} \cdots R_{2 k}\right) \cdots B_{k}(u-k+1) .
$$

This expression is as an element of End $\left(\mathbb{C}^{n}\right)^{\otimes k} \otimes \mathcal{B}(n, l)\left[\left[u^{-1}\right]\right]$.

Let $A_{n}$ be the full anti-symmetriztion operator in the space End $\left(\mathbb{C}^{n}\right)^{\otimes n}$ :

$$
A_{n}\left(v_{1} \otimes \cdots \otimes v_{n}\right)=\sum_{\sigma \in S_{n}}(-1)^{\sigma} v_{\sigma(1)} \otimes \cdots \otimes v_{\sigma(n)} .
$$

One can show [5] that $A_{n}\left\langle B_{1}, \ldots, B_{n}\right\rangle$ is equal to the product of the anti-symmetrizer $A_{n}$ and a series in $u^{-1}$ with coefficients in $\mathcal{B}(n, l)$. This series is called Sklyanin determinant. 
Definition 1. Sklyanin determinant of $B(u)$ is such a series in $u^{-1}$ with coefficients in $\mathcal{B}(n, l)$ that

$$
A_{n}\left\langle B_{1}, \ldots, B_{n}\right\rangle=\operatorname{sdet} B(u) A_{n} .
$$

Proposition 1 ([5]). The coefficients of the series $\operatorname{sdet} B(u)$ are central in $\mathcal{B}(n, l)$. The odd coefficients $c_{1}, c_{3}, \ldots$ in the expansion

$$
\operatorname{sdet} B(u)=(-1)^{l}+c_{1} u^{-1}+c_{2} u^{-2}+c_{3} u^{-3}+\cdots
$$

are algebraically independent generators of the center of $\mathcal{B}(n, l)$.

\section{Alternative definition of the Sklyanin determinant}

The following proposition allows to simplify the combinatorial description of sdet $B(u)$.

Proposition 2. For $k=1, \ldots, n$, define

$$
\Pi_{k}:=1-(2 u-k-n+2)^{-1} \sum_{i=k+1}^{n} P_{k i},
$$

and put

$$
\left\langle\left\langle B_{1}, \ldots, B_{n}\right\rangle\right\rangle:=B_{1}(u) \Pi_{1} B_{2}(u-1) \Pi_{2} \cdots B_{n-1}(u-n+2) \Pi_{n-1} B_{n}(u-n+1) .
$$

Then

$$
A_{n}\left\langle\left\langle B_{1}, \ldots, B_{n}\right\rangle\right\rangle=\operatorname{sdet} B(u) A_{n} .
$$

Proof. We use the notation $A_{k}$ for the antisymmetrizator in $\left(\mathbb{C}^{n}\right)^{\otimes n}$ that acts on the first $k$ copies of $\mathbb{C}^{n}$, and $A_{n-k}^{\prime}$ for the antisymmetrizator in $\left(\mathbb{C}^{n}\right)^{\otimes n}$ that acts on the last $(n-k)$ copies of $\mathbb{C}^{n}$ :

$$
\begin{aligned}
& A_{k}\left(v_{1} \otimes \cdots \otimes v_{n}\right)=\sum_{\sigma \in S_{k}}(-1)^{\sigma} v_{\sigma(1)} \otimes \cdots \otimes v_{\sigma(k)} \otimes v_{k+1} \otimes \cdots \otimes v_{n}, \\
& A_{n-k}^{\prime}\left(v_{1} \otimes \cdots \otimes v_{n}\right)=\sum_{\sigma \in S_{n-k}}(-1)^{\sigma} v_{1} \otimes \cdots \otimes v_{k} \otimes v_{\sigma(k+1)} \otimes \cdots \otimes v_{\sigma(n)} .
\end{aligned}
$$

\section{Lemma 1.}

$$
A_{n-k}^{\prime} R_{k k+1} \cdots R_{k n}=A_{n-k}^{\prime} \Pi_{k}=\Pi_{k} A_{n-k}^{\prime} .
$$

Proof. We follow the lines of the proof of Proposition 1.6.2 in [1]. Let $k+1 \leq i_{1}<i_{2}<\cdots<$ $i_{m} \leq n$. Then

$$
\left(k, i_{1}\right)\left(k, i_{2}\right) \cdots\left(k, i_{m}\right)=\left(i_{1}, i_{2}\right)\left(i_{1}, i_{3}\right) \cdots\left(i_{1}, i_{m}\right)\left(k, i_{1}\right)
$$

where $(a, b)$ denotes a transposition. Therefore,

$$
A_{n-k}^{\prime} P_{k i_{1}} P_{k i_{2}} \cdots P_{k i_{m}}=(-1)^{m-1} A_{n-k}^{\prime} P_{k i_{1}} .
$$

The product of $R$-matrices $R_{k k+1} \cdots R_{k n}$ is a sum of the identity operator and of monomials $P_{k i_{1}} P_{k i_{2}} \cdots P_{k i_{m}}$ with rational coefficients of the form

$$
\frac{(-1)^{m}}{\left(2 u-i_{1}-k+2\right) \cdots\left(2 u-i_{m}-k+2\right)} .
$$


Using (4), we substitute each monomial by $P_{k i_{1}}$ and collect the coefficients for each of $i=$ $k+1, \ldots, n$. Then the first equality of the lemma follows.

In the group algebra of the symmetric group $S_{n}$ for any $i, j, k \in\{1, \ldots, n\}$, such that $i, j, k$ are pairwise distinct numbers, one has

$$
(i, j)((k, i)+(k, j))=((k, i)+(k, j))(i, j) .
$$

Since $A_{n-k}^{\prime}$ is a linear combination of products of transpositions $P_{i j}$ with $i>k$ and $j>k$, by (5) the antisymmetrizer $A_{n-k}^{\prime}$ commutes with the sum $\sum_{i=k+1}^{n} P_{k i}$, and the second equality follows.

For $i \leq m \leq n$ the antisymmetrizer $A_{n-m}^{\prime}$ commutes with $B_{i}(u-i+1)$, and by (3) it also commutes with $\Pi_{m}$. Using that for $m=2,3, \ldots, n$,

$$
(m-1) ! A_{m}^{\prime}=A_{m}^{\prime} A_{m-1}^{\prime},
$$

and Lemma 1, we can rewrite the product $A_{n}\left\langle B_{1}, \ldots, B_{n}\right\rangle$ in the definition of sdet $B(u)$ in terms of operators $\Pi_{1}, \ldots, \Pi_{n}$ :

$$
\begin{aligned}
A_{n}\left\langle B_{1}, \ldots, B_{n}\right\rangle & =\prod_{i=1}^{n-1} \frac{1}{(n-i) !} A_{n} B_{1}(u) \Pi_{1} A_{n-1}^{\prime} B_{2}(u-1) \Pi_{2} \ldots A_{1}^{\prime} B_{n}(u-n+1) \\
& =A_{n} B_{1}(u) \Pi_{1} B_{2}(u-1) \Pi_{2} \cdots B_{n}(u-n+1)=A_{n}\left\langle\left\langle B_{1}, \ldots, B_{n}\right\rangle\right\rangle .
\end{aligned}
$$

\section{Properties of $\left\langle\left\langle B_{1}, \ldots, B_{n}\right\rangle\right\rangle$}

Proposition 2 states that Sklyanin determinant can be computed using the antisymmetrized product $\left\langle\left\langle B_{1}, \ldots, B_{n}\right\rangle\right\rangle$ instead of the antisymmetrized product $\left\langle B_{1}, \ldots, B_{n}\right\rangle$. In this section we give three combinatorial descriptions of the product $\left\langle\left\langle B_{1}, \ldots, B_{n}\right\rangle\right\rangle$.

Let $I_{n} \subset \mathbb{Z}^{n}$ be the subset of such $n$-tuples $\kappa=\left(k_{1}, \ldots, k_{n}\right)$ that

$$
i \leq k_{i} \leq n \quad \text { for all } \quad i=1,2, \ldots, n \text {. }
$$

For $\kappa \in I_{n}$ introduce a rational function $\alpha(\kappa)=\alpha(\kappa, u)$ of a variable $u$ defined by

$$
\alpha(\kappa)=\prod_{i<k_{i}} \frac{1}{(n-2 u-2+i)} .
$$

For all $k=1, \ldots, n$ we identify $P_{k k}$ with $\operatorname{Id}^{\otimes n} \in \operatorname{End}\left(\mathbb{C}^{n}\right)^{\otimes n}$. Then

$$
\left\langle\left\langle B_{1}, \ldots, B_{n}\right\rangle\right\rangle=\sum_{\kappa=\left(k_{1}, \ldots, k_{n}\right) \in I_{n}} \alpha(\kappa) B_{1}(u) P_{1 k_{1}} \cdots B_{n}(u-n+1) P_{n k_{n}}
$$

(The last operator $P_{n k_{n}}=P_{n n}=\mathrm{Id}^{\otimes n}$ in the end of the formula is added for a uniform presentation.)

Lemma 2. For any $X \in \operatorname{End}\left(\mathbb{C}^{n}\right) \otimes \mathcal{B}(n, l)$

$$
P_{i j} X_{i}=X_{j} P_{i j}, \quad X_{i} P_{i j}=P_{i j} X_{j}
$$

Using this simple fact all permutation operators in the expression $\left\langle\left\langle B_{1}, \ldots, B_{n}\right\rangle\right\rangle$ can be moved in front of all elements $B_{s}(u-a)$. 
Proposition 3. Put

$$
Q_{\kappa}^{-1}=P_{1 k_{1}} \cdots P_{n k_{n}}
$$

and denote the corresponding element of symmetric group $S_{n}$ as

$$
q_{\kappa}=\left(n, k_{n}\right)\left(n-1, k_{n-1}\right) \cdots\left(1, k_{1}\right) \in S_{n} .
$$

Define also

$$
q_{\kappa}^{[i]}=\left(n, k_{n}\right)\left(n-1, k_{n-1}\right) \cdots\left(i, k_{i}\right) \in S_{n} .
$$

Then

$$
\left\langle\left\langle B_{1}, \ldots, B_{n}\right\rangle\right\rangle=\sum_{\kappa \in I_{n}} \alpha(\kappa) Q_{\kappa}^{-1} B_{q_{\kappa}^{[1]}(1)}(u) \cdots B_{q_{\kappa}^{[n]}(n)}(u-n+1) .
$$

Here $q_{\kappa}^{[i]}(j)$ is the result of action of permutation $q_{\kappa}^{[i]}$ on $j$.

Proof. From Lemma 2 and the equality (6) the statement of the proposition follows immediately.

For the second combinatorial description of $\left\langle\left\langle B_{1}, \ldots, B_{n}\right\rangle\right\rangle$ we assign to each $\eta \in I_{n}$ a permutation $p_{\eta}$ that is defined by the following recursive rule.

Define a sequence of permutations $\left\{p_{\eta}^{[i]}, i=1, \ldots, n\right\}$ by

$$
p_{\eta}^{[n]}:=\mathrm{Id}, \quad p_{\eta}^{[i]}:=p_{\eta}^{[i+1]}\left(i,\left(p_{\eta}^{[i+1]}\right)^{-1}\left(\eta_{i}\right)\right) \quad \text { for } \quad i=n-1, n-2, \ldots, 1 .
$$

Put $p_{\eta}:=p_{\eta}^{[1]}$.

Proposition 4. Then

$$
\left\langle\left\langle B_{1}, \ldots, B_{n}\right\rangle\right\rangle=\sum_{\eta \in I_{n}} \alpha(\eta) p_{\eta}^{-1} B_{\eta_{1}}(u) \cdots B_{\eta_{n}}(u-n+1),
$$

where $p_{\eta}^{-1}$ is an element of the symmetric group $S_{n}$, identified with the corresponding operator acting on $\left(\mathbb{C}^{n}\right)^{\otimes n}$.

Proof. Consider $\kappa=\left(k_{1}, \ldots, k_{n}\right) \in I_{n}$. Put

$$
\eta=\eta(\kappa)=\left(\eta_{1}, \ldots, \eta_{n}\right)
$$

with

$$
\eta_{i}:=q_{\kappa}^{[i]}(i)=\left(n, k_{n}\right) \cdots\left(i, k_{i}\right)(i) \quad \text { for } \quad i=1, \ldots, n,
$$

where $q_{\kappa}^{[i]}$ is from Proposition 3. Then obviously $\eta_{i} \geq i$ for all $i=1, \ldots, n$, so $\eta=\left(\eta_{1}, \ldots, \eta_{n}\right) \in I_{n}$. Moreover, any $\eta \in I_{n}$ can be obtained from some $\kappa \in I_{n}$ by the formula (8): given $\eta=$ $\left(\eta_{1}, \ldots, \eta_{n}\right) \in I_{n}$, define such $\kappa=\left(k_{1}, \ldots, k_{n}\right)$ recursively:

$$
k_{n}:=\eta_{n}, \quad k_{i}:=\left(i+1, k_{i+1}\right) \cdots\left(n, k_{n}\right)\left(\eta_{i}\right) \quad \text { for } \quad i=n-1, \ldots, 2,1 .
$$

Therefore, the map $\kappa \mapsto \eta(\kappa)$ is a bijection from $I_{n}$ to $I_{n}$. Set $p_{\eta(\kappa)}:=q_{\kappa}$, and observe that $\alpha(\kappa)=\alpha(\eta(\kappa))$. Changing the index of summation $\kappa \in I_{n}$ to $\eta \in I_{n}$ in (7) proves the statement of the Proposition 4. 
In Propositions 3, 4 the sum of monomials is taken over the set $I_{n}$. This set has $n$ ! elements. One can ask, if the monomials can be naturally numerated by permutations $\sigma \in S_{n}$.

We will need the following notion of a word restriction of a permutation. Let $\sigma$ be a permutation of the elements of the set $\{1, \ldots, n\}$, and let $\sigma=\Gamma_{1} \cdots \Gamma_{l}$ be a decomposition into non-intersecting cycles $\Gamma_{i}=\left(g_{i, 1}, \ldots, g_{i, s_{i}}\right)$. Let $G$ be a subset of $\{1, \ldots, n\}$.

Definition 2. We say that the cycle $\left.\Gamma\right|_{G}$ is the word restriction of the cycle $\Gamma$ on the set $G$, if $\left.\Gamma\right|_{G}$ is obtained from $\Gamma$ by deleting from the word of the cycle of $\Gamma$ of all the elements that do not belong to $G$. Then the word restriction of $\sigma$ on the set $G$ is defined as a product of word restrictions of cycles $\left.\Gamma_{1}\right|_{G}, \ldots,\left.\Gamma_{l}\right|_{G}$ on the set $G$ :

$$
\left.\sigma\right|_{G}=\left.\left.\Gamma_{1}\right|_{G} \cdots \Gamma_{l}\right|_{G}
$$

We say that a cycle $\Gamma$ has an empty restriction on the set $G$, if it contains no elements from $G$.

Example 1. The word restriction of the permutation $\sigma=(1,5,7,3)(4)(6,2)$ of the set $\{1,2,3,4$, $5,6,7\}$ on the subset $G=\{1,2,3\}$ is the permutation $(1,3)(2)$.

Proposition 5. For any $\sigma \in S_{n}$ consider a decomposition of $\sigma$ into nonintersecting cycles: $\sigma=\Gamma_{1} \cdots \Gamma_{s}$. Let $g_{i}$ be the maximal element of the cycle $\Gamma_{i}$. Denote as $G_{\sigma}$ the set $\left\{g_{i}\right\}_{i=1}^{s}$. Then

$$
\left\langle\left\langle B_{1}, \ldots, B_{n}\right\rangle\right\rangle=\sum_{\sigma \in S_{n}} \bar{\alpha}(\sigma) \sigma^{-1} B_{\sigma^{[1]}(1)}(u) \cdots B_{\sigma^{[n]}(n)}(u-n+1) .
$$

Here

$$
\bar{\alpha}(\sigma)=\prod_{g \notin G_{\sigma}} \frac{1}{(n-2 u-2+g)},
$$

the permutation $\sigma^{[i]}$ is the word restriction of $\sigma$ on the set $\{i, \ldots, n\}$. We identify $\sigma^{-1}$ with the corresponding operator acting on $\left(\mathbb{C}^{n}\right)^{\otimes n}$.

Proof. Observe that any $\sigma \in S_{n}$ can be written uniquely as a product of transpositions

$$
\sigma=\left(n, k_{n}\right) \cdots\left(1, k_{1}\right)
$$

for some $\kappa=\left(k_{1}, \ldots, k_{n}\right) \in I_{n}$. In other words, there is a one-to-one correspondence between the elements of $I_{n}$ and permutations in $S_{n}$. One can check that the word restriction of $\sigma$ on $\{i, \ldots, n\}$ has the property

$$
\sigma^{[i]}=\left(n, k_{n}\right) \cdots\left(i, k_{i}\right)=q_{\kappa}^{[i]} .
$$

The set of maximal elements of the cycles $\left\{g_{i}\right\}_{i=1}^{s}$ in the decomposition of $\sigma$ into non-intersecting cycles coincides with the set $\left\{k_{i} \mid k_{i}=i\right\}$ in the decomposition (9). This implies the formula for $\bar{\alpha}(\sigma)$.

\section{Computation of Sklyanin determinant}

In this section we give formulas that allow to compute explicitly Sklyanin determinant in terms of matrix elements of the generating matrix $B(u)$.

For any $n$-tuple $\eta \in I_{n}$ we will associate several quantities and sets. The $n$-tuple $\eta \in I_{n}$ can be considered as a function on the interval of integer numbers $\{1, \ldots, n\}$. If $\eta=\left(\eta_{1}, \ldots, \eta_{n}\right)$, then

$$
\eta: \quad\{1, \ldots, n\} \rightarrow\{1, \ldots, n\}, \quad \eta(i):=\eta_{i} .
$$


Let the image $\operatorname{Im}(\eta)$ consist of distinct numbers $\left\{N_{1}, \ldots, N_{K}\right\}$, and let the pre-image $\eta^{-1}\left(N_{l}\right)=$ $\left\{i \mid \eta_{i}=N_{l}\right\}$ consist of numbers $\left\{a_{l, 1}, \ldots, a_{l, m_{l}}\right\}$. We choose the order of the elements so that

$$
a_{l, 1}<\cdots<a_{l, m_{l}} .
$$

For each $l=1, \ldots, K$ the sequence $a_{l, 1}<\cdots<a_{l, m_{l}}$ defines a increasing cyclic permutation $\Gamma_{l}=\left(a_{l, 1}, \ldots, a_{l, m_{l}}\right) \in S_{n}$. Put

$$
\gamma_{\eta}=\Gamma_{1} \cdots \Gamma_{K}
$$

Thus, for every $\eta \in I_{n}$ we associate a permutation $\gamma_{\eta} \in S_{n}$ that is a product of non-intersecting increasing cycles. We also denote as $G_{\eta}^{ \pm}$the sets of maximal and minimal elements of those cycles:

$$
G_{\eta}^{-}=\left\{a_{l, 1}\right\}_{l=1}^{K}, \quad G_{\eta}^{+}=\left\{a_{l, m_{l}}\right\}_{l=1}^{K} .
$$

Let $S(\eta)$ be the the subgroup of $S_{n}$ of all permutations that act trivially on the complement of the set $\operatorname{Im}(\eta)$ in $\{1, \ldots, n\}$.

Consider the set $\left\{b_{q}^{p}(u)\right\}_{p, q=1, \ldots, n}$ of matrix coefficients of $B(u)$ and the set of matrix elements $\left\{b_{p_{1}, \ldots, p_{n}}^{q_{1}, \ldots, q_{n}}(u, \eta)\right\}_{p_{i}, q_{i}=1, \ldots, n}$ of $B_{\eta_{1}}(u) \cdots B_{\eta_{n}}(u-n+1)$ :

$$
\begin{aligned}
& B(u)=\sum_{p, q=1, \ldots, n} E_{p q} \otimes b_{p}^{q}(u), \\
& B_{\eta_{1}}(u) \cdots B_{\eta_{n}}(u-n+1)=\sum_{p_{1}, \ldots, p_{n}, q_{1}, \ldots, q_{n}} E_{p_{1} q_{1}} \otimes \cdots \otimes E_{p_{n} q_{n}} b_{p_{1}, \ldots, p_{n}}^{q_{1}, \ldots, q_{n}}(u, \eta) .
\end{aligned}
$$

\section{Theorem 1.}

sdet $B(u)=\sum_{\eta \in I_{n}} \sum_{\sigma \in S(\eta)} \alpha(\sigma, \eta) b_{\sigma(1), \ldots, \sigma(n)}^{1, \ldots, n}(u, \eta)$,

where $b_{\sigma(1), \ldots, \sigma(n)}^{1, \ldots, n}(u, \eta)$ is the corresponding matrix coefficient of $B_{\eta_{1}}(u) \cdots B_{\eta_{n}}(u-n+1)$. It can be computed by the following rule:

$$
\begin{aligned}
& b_{\sigma(1), \ldots, \sigma(n)}^{1, \ldots, n}(u, \eta)=\prod_{k=1}^{n} b_{p(k)}^{q(k)}(u-k+1), \\
& p(k)=\left\{\begin{array}{ll}
\sigma(\eta(k)) & \text { if } k \in G_{\eta}^{-}, \\
s_{k} & \text { otherwise, }
\end{array} \quad q(k)= \begin{cases}\eta(k) & \text { if } k \in G_{\eta}^{+}, \\
s_{\gamma_{\eta}(k)} & \text { otherwise, }\end{cases} \right.
\end{aligned}
$$

and the Einstein summation convention is used in (10) for the indices $s_{t}$ : if for some $t$ the repeated indices $s_{t}$ occur in (10), the monomials are implicitly summed over the range $s_{t}=$ $1, \ldots, n$. Also

$$
\alpha(\sigma, \eta)=(-1)^{\sigma} \prod_{i<\eta_{i}} \frac{1}{(2 u+2-i-n)} .
$$

Proof. From Proposition 4 we can write

$$
\operatorname{sdet} B(u) A_{n}=\left(\sum_{\eta \in I_{n}} A_{n} \alpha(\eta)(-1)^{p_{\eta}} B_{\eta_{1}}(u) \cdots B_{\eta_{n}}(u-n+1)\right) .
$$


Following the lines of the proof ${ }^{1}$ of Proposition 2.7 in [4] we apply both sides of (11) to the vector $\left(e_{1} \otimes \cdots \otimes e_{n}\right)$. Let $v=A_{n}\left(e_{1} \otimes \cdots \otimes e_{n}\right)$. The left-hand side of (11) gives sdet $B(u) v$. The right-hand side equals

$$
\sum_{\eta \in I_{n}} A_{n} \alpha(\eta)(-1)^{p_{\eta}} \sum_{t_{1}, \ldots, t_{n}} b_{t_{1}, \ldots, t_{n}}^{1, \ldots, n}(u, \eta)\left(e_{t_{1}} \otimes \cdots \otimes e_{t_{n}}\right) .
$$

If not all of $\left\{t_{1}, \ldots, t_{n}\right\}$ are pairwise distinct, then $A_{n}\left(e_{t_{1}} \otimes \cdots \otimes e_{t_{n}}\right)=0$. Otherwise $e_{t_{1}} \otimes \cdots \otimes$ $e_{t_{n}}=\sigma\left(e_{1} \otimes \cdots \otimes e_{n}\right)$, where the permutation $\sigma \in S_{n}$ is defined by $\sigma(k)=t_{k}$, and

$$
A_{n}\left(e_{t_{1}} \otimes \cdots \otimes e_{t_{n}}\right)=A_{n} \sigma\left(e_{1} \otimes \cdots \otimes e_{n}\right)=(-1)^{\sigma} v .
$$

We obtain that

$$
\begin{gathered}
\sum_{\eta \in I_{n}} A_{n} \alpha(\eta)(-1)^{p_{\eta}} \sum_{t_{1}, \ldots, t_{n}} b_{t_{1}, \ldots, t_{n}}^{1, \ldots, n}(u, \eta)\left(e_{t_{1}} \otimes \cdots \otimes e_{t_{n}}\right) \\
=\sum_{\sigma \in S_{n}} \sum_{\eta \in I_{n}} \alpha(\eta)(-1)^{p_{\eta}}(-1)^{\sigma} b_{\sigma(1), \ldots, \sigma(n)}^{1, \ldots, n}(u, \eta) v .
\end{gathered}
$$

Observe that if for fixed $\sigma \in S_{n}$ there exists $j \notin \operatorname{Im} \eta$ such that $\sigma(j) \neq j$, then the corresponding matrix element $b_{\sigma(1), \ldots, \sigma(n)}^{1, \ldots, n}(u, \eta)$ vanishes to zero. Hence, we can substitute the sum in (12) over all permutations $\sigma \in S_{n}$ by the sum of permutations $\sigma \in S(\eta)$ and rewrite (12) as

$$
\sum_{\eta \in I_{n}} \sum_{\sigma \in S(\eta)} \alpha(\eta)(-1)^{p_{\eta}}(-1)^{\sigma} b_{\sigma(1), \ldots, \sigma(n)}^{1, \ldots, n}(u, \eta) v .
$$

The direct check shows that $\alpha(\sigma, \eta)=\alpha(\eta)(-1)^{p_{\eta}}(-1)^{\sigma}$ and that the matrix coefficients $b_{\sigma(1), \ldots, \sigma(n)}^{1, \ldots, n}$ can be computed by (10).

\section{Examples: $n=2,3$}

The Theorem 1 allows to compute Sklyanin determinant explicitly.

Example 2. For $n=2$ the set $I_{2}$ has two elements: $I_{2}=\{(1,2),(2,2)\}$. Then $\eta=(1,2)$ contributes

$$
b_{1}^{1}(u) b_{2}^{2}(u-1)-b_{2}^{1}(u) b_{1}^{2}(u-1),
$$

and $\eta=(2,2)$ contributes

$$
\frac{1}{2 u-1}\left(b_{2}^{1}(u) b_{1}^{2}(u-1)+b_{2}^{2}(u) b_{2}^{2}(u-1)\right),
$$

so sdet $B(u)$ equals

$$
b_{1}^{1}(u) b_{2}^{2}(u-1)-\frac{2 u-2}{2 u-1} b_{2}^{1}(u) b_{1}^{2}(u-1)+\frac{1}{2 u-1} b_{2}^{2}(u) b_{2}^{2}(u-1) .
$$

Example 3. For $n=3$ the set $I_{3}$ has six elements. They make the following contributions to sdet $B(u)$ :

$$
\eta=(1,2,3) \quad \sum_{\sigma \in S_{3}}(-1)^{\sigma} b_{\sigma(1)}^{1}(u) b_{\sigma(2)}^{2}(u-1) b_{\sigma(3)}^{3}(u-2),
$$

\footnotetext{
${ }^{1}$ The author would like to thank the referee for indicating this step.
} 


$$
\begin{aligned}
& \eta=(2,2,3) \quad \frac{1}{2 u-2} \sum_{s=1}^{3} b_{2}^{s}(u) b_{s}^{2}(u-1) b_{3}^{3}(u-2)-b_{3}^{s}(u) b_{s}^{2}(u-1) b_{2}^{3}(u-2), \\
& \eta=(3,2,3) \quad \frac{1}{2 u-2} \sum_{s=1}^{3} b_{3}^{s}(u) b_{2}^{2}(u-1) b_{s}^{3}(u-2)-b_{2}^{s}(u) b_{3}^{2}(u-1) b_{s}^{3}(u-2), \\
& \eta=(1,3,3) \quad \frac{1}{2 u-3} \sum_{s=1}^{3} b_{1}^{1}(u) b_{3}^{s}(u-1) b_{s}^{3}(u-2)-b_{3}^{1}(u) b_{1}^{s}(u-1) b_{s}^{3}(u-2), \\
& \eta=(2,3,3) \quad \frac{1}{(2 u-2)(2 u-3)} \sum_{s=1}^{3} b_{2}^{2}(u) b_{3}^{s}(u-1) b_{s}^{3}(u-2)-b_{3}^{2}(u) b_{2}^{s}(u-1) b_{s}^{3}(u-2), \\
& \eta=(3,3,3) \quad \frac{1}{(2 u-2)(2 u-3)} \sum_{s, t=1}^{3} b_{3}^{s}(u) b_{s}^{t}(u-1) b_{t}^{3}(u-2) .
\end{aligned}
$$

The value of sdet $B(u)$ equals the sum of these six expressions. We introduce the notation

$$
\left|\begin{array}{ccc}
k & l & m \\
p & r & t
\end{array}\right|:=b_{p}^{k}(u) b_{r}^{l}(u-1) b_{t}^{m}(u-2) \text {. }
$$

Then

$$
\begin{aligned}
\operatorname{sdet} & B(u)=\left|\begin{array}{lll}
1 & 2 & 3 \\
1 & 2 & 3
\end{array}\right|+\frac{1}{2 u-2}\left(\left|\begin{array}{lll}
2 & 2 & 3 \\
2 & 2 & 3
\end{array}\right|+\left|\begin{array}{lll}
3 & 2 & 3 \\
3 & 2 & 3
\end{array}\right|-\left|\begin{array}{lll}
1 & 1 & 3 \\
3 & 1 & 1
\end{array}\right|-\left|\begin{array}{lll}
1 & 3 & 3 \\
3 & 1 & 3
\end{array}\right|\right) \\
& +\frac{1}{2 u-3}\left(\left|\begin{array}{lll}
1 & 1 & 3 \\
1 & 3 & 1
\end{array}\right|+\left|\begin{array}{lll}
1 & 3 & 3 \\
1 & 3 & 3
\end{array}\right|\right)-\frac{2 u-3}{2 u-2}\left(\left|\begin{array}{lll}
1 & 2 & 3 \\
2 & 1 & 3
\end{array}\right|+\left|\begin{array}{lll}
1 & 2 & 3 \\
3 & 2 & 1
\end{array}\right|-\left|\begin{array}{lll}
1 & 2 & 3 \\
2 & 3 & 1
\end{array}\right|\right) \\
& -\frac{2 u-4}{2 u-3}\left|\begin{array}{lll}
1 & 2 & 3 \\
1 & 3 & 2
\end{array}\right|-\frac{2 u-4}{2 u-2}\left|\begin{array}{ccc}
1 & 2 & 3 \\
3 & 1 & 2
\end{array}\right|+\frac{2 u-4}{(2 u-2)(2 u-3)}\left(\left|\begin{array}{lll}
2 & 2 & 3 \\
2 & 3 & 2
\end{array}\right|+\left|\begin{array}{lll}
3 & 2 & 3 \\
3 & 3 & 2
\end{array}\right|\right) \\
& +\frac{1}{(2 u-2)(2 u-3)}\left(\left|\begin{array}{lll}
2 & 1 & 3 \\
2 & 3 & 1
\end{array}\right|+\left|\begin{array}{lll}
2 & 3 & 3 \\
2 & 3 & 3
\end{array}\right|+\left|\begin{array}{lll}
3 & 1 & 3 \\
3 & 3 & 1
\end{array}\right|+\left|\begin{array}{lll}
3 & 3 & 3 \\
3 & 3 & 3
\end{array}\right|\right) .
\end{aligned}
$$

\section{Acknowledgements}

The author would like to thank A.I. Molev and the referees for valuable comments and suggestions that allowed to simplify significantly the formulas and to improve the text of the paper. The travel to RAQIS'10 was supported by AWM travel grant. The research is partially supported by KSU Mentoring fellowship for WMSE.

\section{References}

[1] Molev A., Yangians and classical Lie algebras, Mathematical Surveys and Monographs, Vol. 143, American Mathematical Society, Providence, RI, 2007.

[2] Molev A., Yangians and their applications, in Handbook of Algebra, Vol. 3, North-Holland, Amsterdam, 2003, 907-959, math.QA/0211288.

[3] Molev A., Sklyanin determinant, Laplace operators, and charcteristic identities for classical Lie algebras, J. Math. Phys. 36 (1995), 923-943, hep-th/9409036.

[4] Molev A., Nazarov M., Ol'shanskii G., Yangians and classical Lie algebras, Russian Math. Surveys 51 (1996), no. 2, 205-282, hep-th/9409025.

[5] Molev A., Ragoucy E., Representations of reflection algebras, Rev. Math. Phys. 14 (2002), 317-342, math.QA/0107213.

[6] Ol'shanskii G.I., Twisted Yangians and infinite-dimensional classical Lie algebras, in Quantum Groups (Leningrad, 1990), Editor P. Kulish, Lecture Notes in Math., Vol. 1510, Springer, Berlin, 1992, $104-119$.

[7] Sklyanin E.K., Boundary conditions for integrable quantum systems, J. Phys. A: Math. Gen. 21 (1988), 2375-2389. 